\title{
Conditional Measurements of Optically-Aberrating Structures in Transonic Attached Boundary Layers
}

\author{
Aaron Buckner* \\ Northrop Grumman Space Technology, One Space Park R1/1062, Redondo Beach, CA, 90278 \\ Stanislav Gordeyev ${ }^{\dagger}$ \\ University of Notre Dame, Notre Dame, IN, 46556 \\ and \\ Eric Jumpert \\ University of Notre Dame, Notre Dame, IN, 46556
}

\begin{abstract}
This paper is a further extension of an investigation of the optical distortions caused by a transonic, attached, turbulent boundary layer reported in earlier paper. Simultaneous, time resolved $x$-wire, surface pressure and optical measurements were performed to investigate the physical mechanism of the optical aberrations. A conditional sampling algorithm, triggered on local minima in the optical path difference, was implemented to extract the flow features of optically aberrating flow structures. The conditionally averaged surface pressure showed a pressure well associated with optically aberrating flow structures. A technique to identify coherent vortical structures is applied to the conditionally averaged velocity field, and it shown that a large scale, coherent, vortical structure resides over the optical measurement location. The conditionally averaged vortical structure extends $\sim \delta^{*}$ in the streamwise direction and $\sim 1.2 \delta^{*}$ in the wall normal direction, where $\delta^{*}$ is the boundary layer displacement thickness.
\end{abstract}

\section{Introduction}

When an otherwise planar optical wavefront is made to propagate through a variable index of refraction, turbulent flow field, the wavefront becomes aberrated, adversely affecting its far-field intensity pattern. This degraded far-field intensity pattern is undesirable for use in optical systems. The study of optical propagation through such flow fields is known as aero-optics ${ }^{1}$. Variable index flow fields come in many varieties, such as a mixing layer between two dissimilar index flow streams, and compressible boundary and free shear layers. The latter two scenarios are of great interest for the use of lasers on airborne platforms, specifically at Mach numbers greater than 0.5 and 0.3 , respectively.

It has been found that the physical mechanism for optical aberrations in a free shear layer are the low pressure wells (and their concomitant density wells) associated with the coherent vortical structures that roll-up naturally in the free shear layer ${ }^{2}$. It has been proposed that a similar mechanism is responsible for the optical aberrations in a turbulent boundary layer.

The concept of hairpin vortices as a main mechanism of producing coherent structures in turbulent boundary layers is well established, see Robinson ${ }^{3}$ review article, for instance. The horseshoe vortex structures were found to occupy the outer part of the boundary layer ${ }^{4}$ and to have a relatively high convective velocity 0.8-0.9 U free, see Ref. 5. Numerically, LES simulations of compressible turbulent

\footnotetext{
* Staff Engineer, Fluid \& Thermodynamics Dept., Member AIAA

${ }^{\dagger}$ Research Assistant Professor, Department of Aerospace and Mech. Eng., Member AIAA

* Professor, Department of Aerospace and Mech. Eng., Fellow AIAA
} 
boundary layers ${ }^{6}$ for Mach number of 0.9 revel the presence of large, on the order of $2 \delta^{*}$, vortical structures in the outer part of the boundary layer, responsible for optical aberrations.

The two parent papers to the current work ${ }^{7,8}$ have investigated optical properties of the turbulent boundary layer and provided a good amount of indirect evidence that the large scale, coherent vortical structures present in a turbulent boundary layer are responsible for the optical aberrations. A high convective velocity ( $80 \%$ of the freestream) was measured for optical aberrations, indicating that these structures reside in the outer portion of the boundary layer. A simple scaling argument, based on the pressure well associated with a 2 dimensional vortex, successfully predicted the functional form onto which the optical data should collapse. A similar scaling argument for the optical aberrations of sheared flows predicted the same functional form. In addition, similar convective speeds were seen in the surface pressures, further indicating that pressure wells associated with coherent, vortical structures with corresponding pressure wells are responsible for the optical aberrations in turbulent boundary layers. The streamwise correlation length of optically active flow structures was measured to be $\sim 2 \delta^{*}$.

To provide direct evidence on the nature of optical distortions in the turbulent boundary layers, simultaneous optical, pressure and velocity measurements were taken in the outer part of the boundary layer. This paper reports the results of conditional analysis of the data.

\section{Experimental Set-up}

All experiments were conducted at the Hessert Laboratory at the University of Notre Dame. The facilities were described in detail in Refs. 7 and 9, and the interested reader is referred to them for a complete discussion. A complete description of measurement techniques and data reduction procedures can be found in Ref. 9. Below we provide a brief description of the experimental set-up.
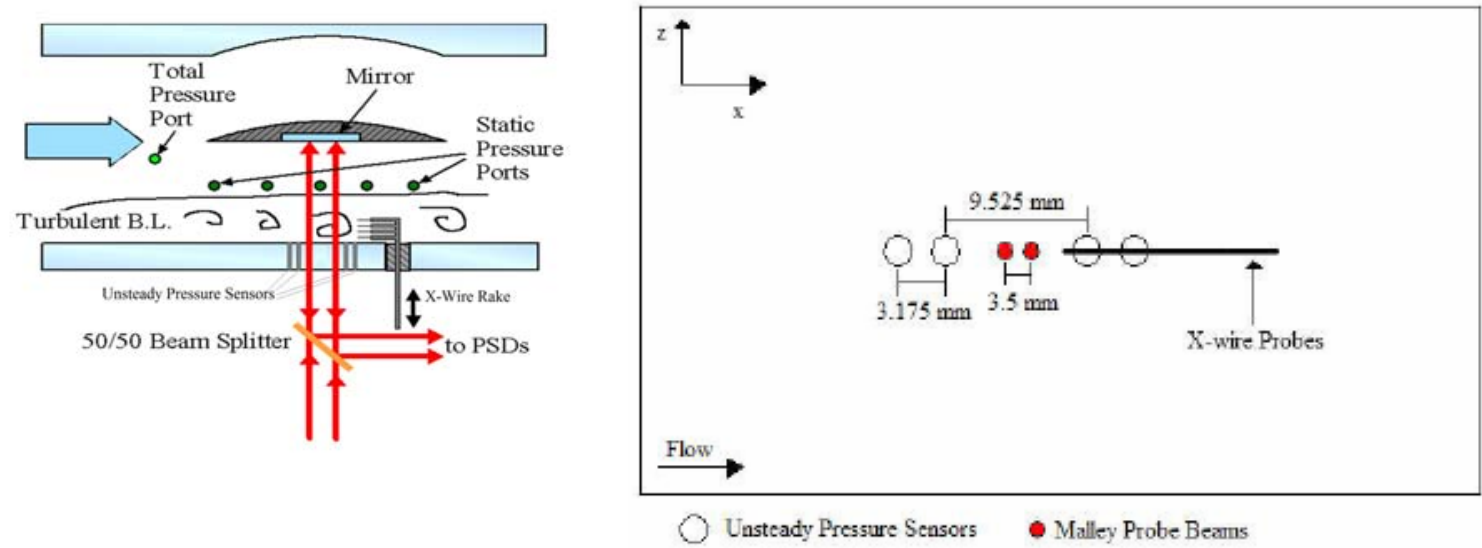

Figure 1. Schematic of the test section: Left: Side View, Right: Floor View.

All of the experiments were conducted in indraft transonic wind tunnels in the Hessert Laboratory at the University of Notre Dame, powered by up to three vacuum pumps. The tunnel section was a square duct with $9.9 \mathrm{~cm} \mathrm{X} 10.1 \mathrm{~cm}$ in cross-section and $170 \mathrm{~cm}$ long and was made of optically transparent Plexiglas. The floor of the first $70 \mathrm{~cm}$ of the tunnel was roughened to enhance the onset and initial growth of the turbulent boundary layer. After this initial roughened section, the boundary layer was allowed to develop naturally. The incoming Mach number was 0.52 for all conditional measurements. The instrumented test section was placed by the end of the tunnel section. Schematics of the test section can be found in Figure 1, and a picture of the test section can be found in Figure 2.

A single hot wire was used to traverse through the flow in the wall normal direction in the test section to measure the boundary layer velocity profile. A $99 \%$ boundary layer thickness $\delta$, displacement thickness $\delta^{*}$ and momentum thicknesses $\theta$ for the boundary layer were calculated to be $2.8 \mathrm{~cm}, 4.5 \mathrm{~mm}$ and $2.5 \mathrm{~mm}$, respectively. The Reynolds number per unit length was $\operatorname{Re}=1.810^{6} 1 / \mathrm{m}$.

Inner variable scaling of the boundary layer revealed that the boundary layer exhibits a well defined log region for $\mathrm{y}^{+} \sim 400 . .6000\left(\mathrm{y} / \delta^{*} \sim 0.02 . .25\right)$, as well as an intermittent region, characterized by

Page 2 of 10

American Institute of Aeronautics and Astronautics 
the velocity approaching a constant value in the free stream for $\mathrm{y}^{+}>6000$. The friction velocity, determined by fitting a straight line to the data in the $\log$ region, was $\mathrm{u}_{\tau}=6.5 \mathrm{~m} / \mathrm{sec}$. Overall, the boundary layer under investigation was found to be a fully developed turbulent boundary layer.

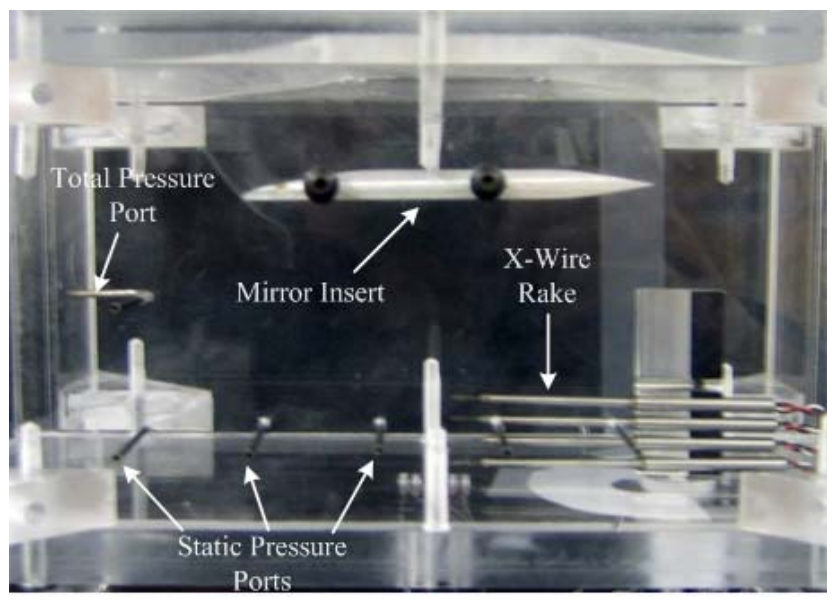

Figure 2. Picture of the test section showing all instrumentation.

The test section was instrumented with static pressure ports on the wall side to monitor flow speed. The floor of the test section was equipped with an array of unsteady pressure sensors shown in Figure 1, right plot. Two pressure sensors were placed upstream of the optical measurement location (marked by two red circles in Figure 1, right plot) and two other pressure sensors were placed downstream of it. A rake of four $\mathrm{x}$-wires, oriented in the wall normal direction with $5 \mathrm{~mm}$ spacing in between probes, was placed just downstream of the optical measurement location to measure the streamwise and wall normal components of velocity.

A streamlined section of aluminum was placed $5.08 \mathrm{~cm}$ above the floor of the test section, and had a mirror installed on its lower side, see Figures 1 and 2. A section of the upper wall was cut out to eliminate blockage effects due to the mirror section. The mirror was used to reflect the optical probe beams back through the boundary layer under investigation. Since the experiment was conducted in a wind tunnel, there was a boundary layer that developed on the top wall in addition to the boundary layer under investigation (that developed on the bottom wall). With the mirror placed in the flow, the optical probe beams only traverse the bottom boundary layer under investigation, so optical measurements can be obtained without ambiguity.

The optical measurement technique used was the Malley probe ${ }^{10,7}$. The Malley probe measures time resolved one-dimensional slices of wavefronts in terms of Optical Path Differences, $\mathrm{OPD}(\mathrm{t})$, using the frozen field approximation. The temporal resolution capability of the sensor is in the order of 100's $\mathrm{MHz}$ and makes it very attractive for this application. A detailed description of the Malley probe and its operation can be found in Reference 7.

All of the data was collected simultaneously at a sampling frequency of $200 \mathrm{kHz}$. This sampling rate was sufficient to avoid temporal aliasing, and the data was high-pass filtered in post processing to remove contamination from mechanical vibrations at low frequencies.

\section{Results}

\section{A. Cross Correlations}

The two previous papers ${ }^{7,8}$ have reported auto-correlation lengths of structures from velocity, surface pressure, and optical measurements. With the simultaneous measurement of optical aberrations, surface pressure, and two components of velocity, it is possible to make cross correlations between the various signals in order to obtain more information about the nature of optical aberrations in a turbulent boundary layer.

The cross correlations are preformed in the frequency domain, with the cross correlation coefficient function defined as

$$
\gamma(f, y)=\frac{\left\langle\hat{s}_{1}(y) \hat{s}_{2}^{*}(y+\Delta y)\right\rangle}{\sqrt{\left\langle\hat{s}_{1}(y) \hat{s}_{1}^{*}(y)\right\rangle\left\langle\hat{s}_{2}(y+\Delta y) \hat{s}_{2}^{*}(y+\Delta y)\right\rangle}}
$$

where $s_{1}(y)$ is the fluctuating component of a particular time series, $s_{2}(y+\Delta y)$ is the fluctuating component of another time series, measured a distance $\Delta y$ away from the first time series. The hat denotes a Fourier transform, the star superscript denotes a complex conjugate, and the brackets denote ensemble 
averaging. This function was used to find the correlation between the optical aberrations OPD and flow parameters such as surface pressure and velocity components.

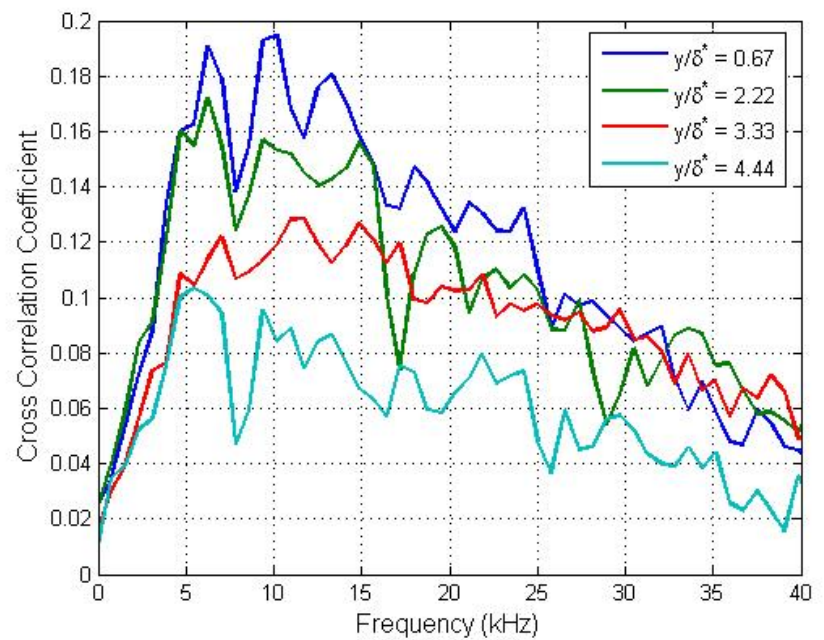

Figure 3. Correlation coefficient function between optical aberrations and streamwise velocity

The correlation function between optical aberrations $\operatorname{OPD}(\mathrm{t})$ and streamwise velocity was computed as a function of wall normal distance (normalized by the boundary layer displacement thickness $\delta^{*}$ ), and is presented in Figure 3. The correlation function for all wall normal distances reaches a maximum value at frequencies $5-10 \mathrm{kHz}$, with the highest value of 0.2 at the wall normal location of $y / \delta^{*}=0.67$. Figure 3 shows that the majority of the correlation between velocity and beam deflection occurs at wall normal distances less than $2 \delta^{*}$, which is consistent the findings in Refs. 7 and 8 . This region is located in the transition region between the log and intermittent or wake regions of the boundary layer, where large scale coherent vortical structures have been found in PIV studies ${ }^{11}$. These PIV findings, coupled with the data presented in Figure 3, indicate that coherent vortical motions in the log to intermittent transition region of the boundary layer are responsible for optical aberrations.

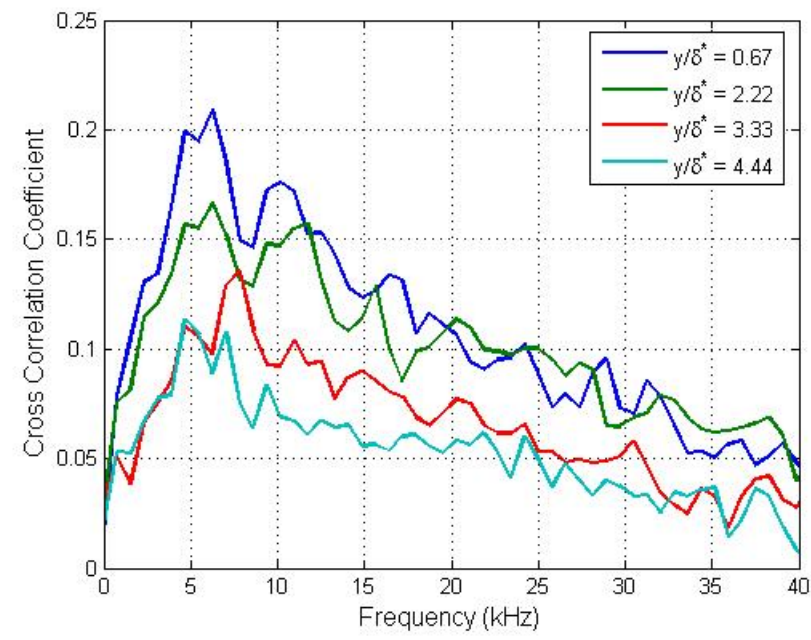

Figure 4. Correlation coefficient function between optical aberrations and wall normal velocity

Correlation functions between $\mathrm{OPD}(\mathrm{t})$ and wall-normal and spanwise velocity components are shown in Figures 4 and 5. These correlations show the maximum correlation values at lower frequency of $5 \mathrm{kHz}$ for all wall-normal location. They also confirm the strongest correlation between OPD and velocity components occur at wall normal distances less than $2 \delta^{*}$. 


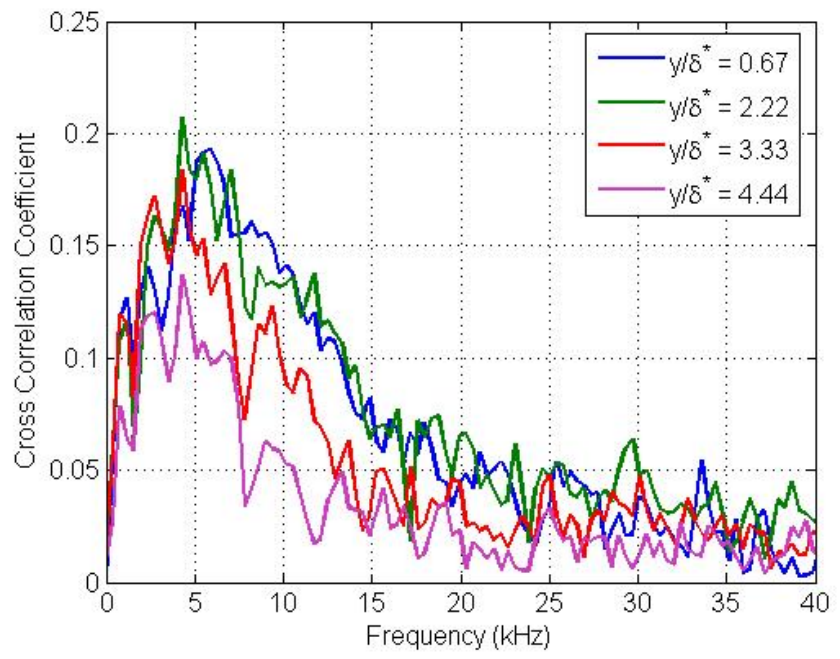

Figure 5. Correlation coefficient function between optical aberrations and spanwise velocity

A characteristic streamwise separation between optical structures can be determined from the correlation coefficient function. The correlation functions between OPD and velocity components have a broad maximum that occurs at frequencies of $5-10 \mathrm{kHz}$. Defining a characteristic streamwise separation between optically active flow structures as

$$
\Lambda_{x}=\frac{U_{c}}{f}
$$

gives a characteristic streamwise separation in the range of $3.3 \delta^{*}$ to $6.8 \delta^{*}$. This length is well correlated with optical separation length of $4 \delta^{*}$, reported in Ref. 10. The broad range of frequencies can be attributed to the fact that velocity and density are not directly related. Optical aberrations are caused by density fluctuations, not velocity fluctuations, so correlating velocity and optical aberrations should not yield a correlation that exhibits a strong peak with a large maximum correlation value.

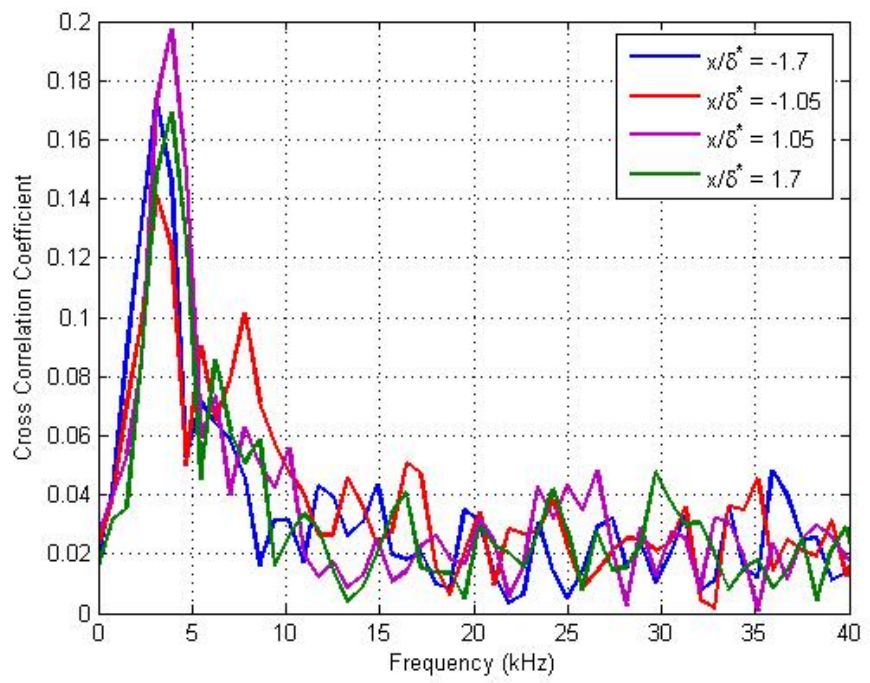

Figure 6. Correlation coefficient function between optical aberrations and surface pressure

The correlation function of Eq. (1) was also used to compute the correlation between optical aberrations OPD and fluctuating surface pressures from all four surface pressure sensors located at different streamwise location relative to the Malley probe position, see Figure 2. The result of this computation can be seen in Figure 6. Since Taylor's hypothesis was assumed to be valid, the cross correlation function in the 
frequency domain should be identical for all of the pressure sensors, which is indeed the case. Correlation functions between OPD and different pressure sensors are very similar in shape with maximum correlation values around 0.2 . Correlation functions show a narrow peak located around $4 \mathrm{kHz}$. Using the characteristic separation length between optically active flow structures defined in Eq. (2), the characteristic separation based on the OPD-pressure correlation was found to be $\sim 8 \delta^{*}$; this is a slightly higher value than the optical separation length determined from OPD-velocity correlation measurements. As pointed out in Ref. 10, there is no reason why the surface pressure should correspond exactly to the vortical structure passing above the surface.

\section{B. Conditional Sampling}

The information reported in the previous section and the two parent papers ${ }^{7,8}$ constitutes indirect evidence that the physical mechanism of optical aberrations in turbulent boundary layers are due to the pressure wells associated with large scale vortical structures in the outer portion of the boundary layer. A conditional sampling algorithm, triggered on local minima in the OPD time series, was implemented to make a direct link between optical aberrations and the flow structure. A pseudo-spatial evolution of $\operatorname{OPD}(\mathrm{x})$ was calculated from $\mathrm{OPD}(\mathrm{t})$ time series using the Taylor's frozen field hypothesis, $\mathrm{x}=-\mathrm{U}_{\mathrm{c}} \mathrm{t}$, where $\mathrm{U}_{\mathrm{c}}=0.8 \mathrm{U}_{\text {freestream }}$ is the convective speed of the optical structure.

Two criteria were used to define the conditional sampling. These two criteria were that the OPD must have a local minimum, and that the value of this local minimum of the OPD must be less than a preset threshold value. Minima of the OPD signal correspond to a drop in the local density, and thus a drop in the local pressure, which is characteristic of a coherent vortical structure. The simultaneous, conditionally sampled OPD, velocity ( $\mathrm{u}$ and $\mathrm{v}$ components) and surface pressure were recorded for 100 time steps before and after the triggering optical event, and an average over few hundred events was calculated over the conditionally sampled ensembles to obtain the conditionally averaged quantities.

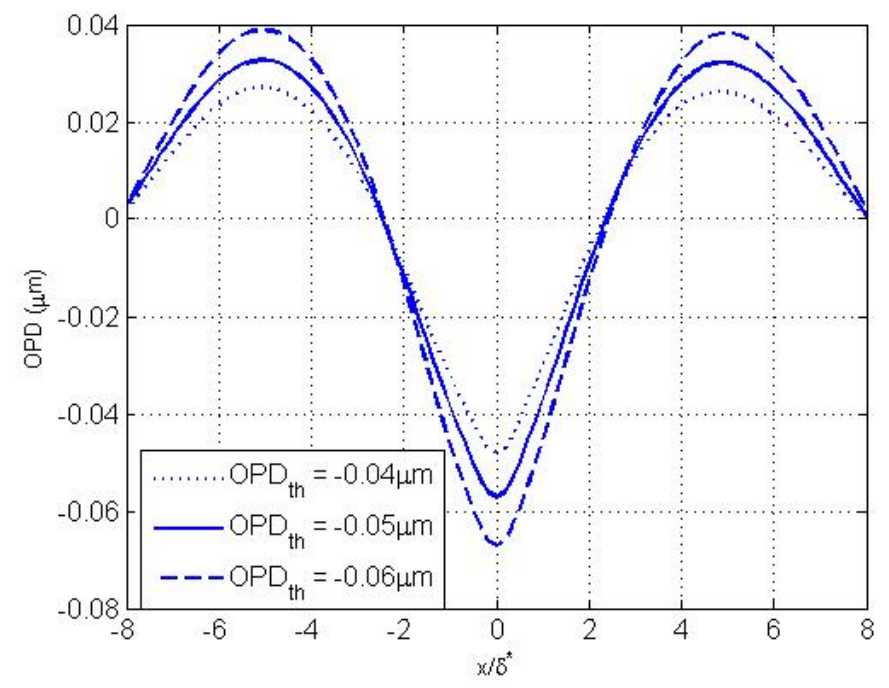

Figure 7. Conditionally averaged OPD for various threshold values

The threshold value for the conditional sampling algorithm was chosen to be $\mathrm{OPD}_{\mathrm{th}}=-0.05 \mu \mathrm{m}$, which was 2.5 times the infinite aperture $\mathrm{OPD}_{\mathrm{rms}}$ of $0.02 \mu \mathrm{m}$ for the freestream Mach number and the local boundary layer thickness. It was found that the resulting conditionally sampled OPD field, averaged over all of the realizations, did not change appreciably with a $20 \%$ increase or decrease in the threshold value from the optimal threshold. The conditionally averaged OPD is shown for the optimal threshold value of $0.05 \mu \mathrm{m}$ is shown in Figure 8, as well as the conditionally averaged OPD for a $20 \%$ change in the threshold value (to $-0.04 \mu \mathrm{m}$ and $-0.06 \mu \mathrm{m}$ ). Figure 8 shows a mild sensitivity to the chosen threshold value, confirming the robustness of the conditional sampling algorithm. The triggering optical event that met the conditional sampling requirements is centered at $\mathrm{x}=0$ in all of the figures to follow.

The pseudo-spatial distance between the two peaks in the conditionally average OPD can be considered as an average streamwise spacing between optically aberrating structures and, from Figure 7, is

Page 6 of 10

American Institute of Aeronautics and Astronautics 
approximately $9 \delta^{*}$. This value is consistent with the streamwise structure spacing of several $\delta^{*}$ found from OPD-velocity/pressure correlation data reported above.

\section{Conditionally Averaged Surface Pressure}

Using the optical conditional triggering described before, the conditionally averaged surface pressures at four streamwise locations were computed and results are shown in Figure 8. If optical aberrations in a turbulent boundary layer are the result of the pressure well associated with large scale, coherent vortical structures in the outer portion of the boundary layer, a pressure well should be present over the optical measurement location $(\mathrm{x}=0)$. Indeed, large scale, although shallow, pressure wells are seen in Figure 8 over the optical measurement location. The streamwise size of the pressure well is on the order of $5 \delta^{*}$, which indicates that these pressure wells are associated with coherent vortical structures in the outer portion of the boundary layer; since the pressure sensors were imbedded in the floor of the test section, sensors were only measuring the "footprint" of the pressure well, which might explain the shallowness of the pressure wells.
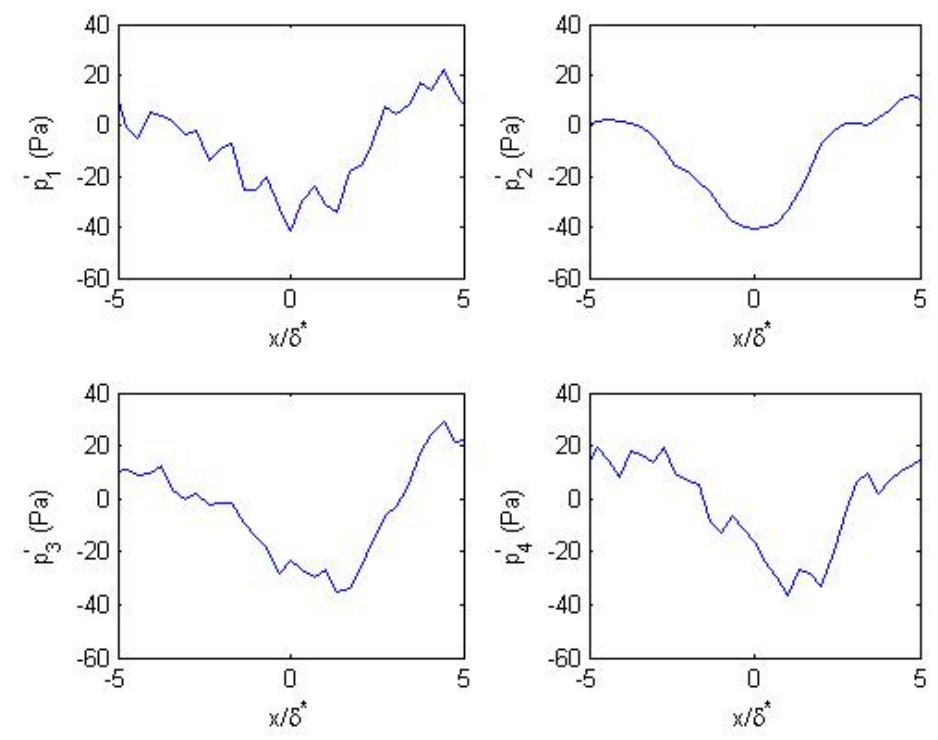

Figure 8. Conditionally averaged surface pressure

\section{Conditionally Averaged Velocity Field}

A rake of four $\mathrm{x}$-wires spanned in the wall-normal direction was used to simultaneously measure two components of velocity. As a result, the conditionally averaged, two dimensional velocity field can be constructed using the conditional triggering form the optical structure. A 2-dimensional velocity field, showing the velocity vectors at the measurement locations, was used to see the signature of related vortical structures for the conditionally triggered optical event. Figure 9 shows the conditionally averaged velocity field, with the pseudo-streamwise coordinate $\mathrm{x}$ been calculated using the frozen convective field assumption. In Figure 9 there appears to be a large scale vortical motion, centered on $\mathrm{x}=0$. The velocity field signature is consistent with that of a vortex centered over the optical measurement location, but this is not sufficient information to conclude that a coherent structure resides at this location. Additionally, it is difficult to determine the spatial extent of a coherent structure from this figure. 


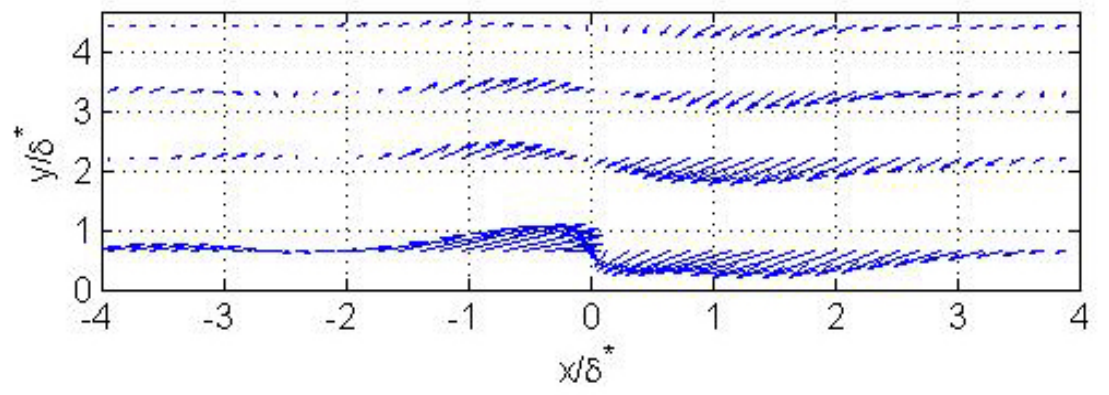

Figure 9. Conditionally averaged velocity field

\section{E. The $-\lambda_{2}$ Criteria}

Jeong and Hussain ${ }^{12}$ have shown that the use of vorticity calculations and pressure well measurements are not the best way to detect the presence of coherent vortical structures. They proposed, with examples, that the proper method of detecting coherent vortical structures is to use the velocity gradient tensor $\mathbf{T}=\left\{\partial u_{i} / \partial x_{j}\right\}$. While local pressure minima cannot be used as a general criterion for the identification of coherent vortical structures, it is a useful starting point in deriving a new criterion.

The new criterion is that a coherent vortical structure is defined as "a connected region with two negative eigenvalues of $S^{2}+\Omega^{2}$ ", where $\mathrm{S}$ is the symmetric portion of the velocity gradient tensor $\mathbf{T}$, and $\Omega$ is the anti-symmetric part of the velocity gradient tensor $\mathbf{T}$. If $\lambda_{1}, \lambda_{2}$, and $\lambda_{3}$ are the eigenvalues, and $\lambda_{1} \geq \lambda_{2} \geq \lambda_{3}$, the criteria simplifies to a connected region with $\lambda_{2}<0$. For planar (two dimensional) flow, there are only two eigenvalues, and this criteria reduces to the smallest eigenvalues being negative. The derivation of this criteria is consistent with a local pressure minima due to coherent vortical motion; however, the $-\lambda_{2}$ criteria is more robust.

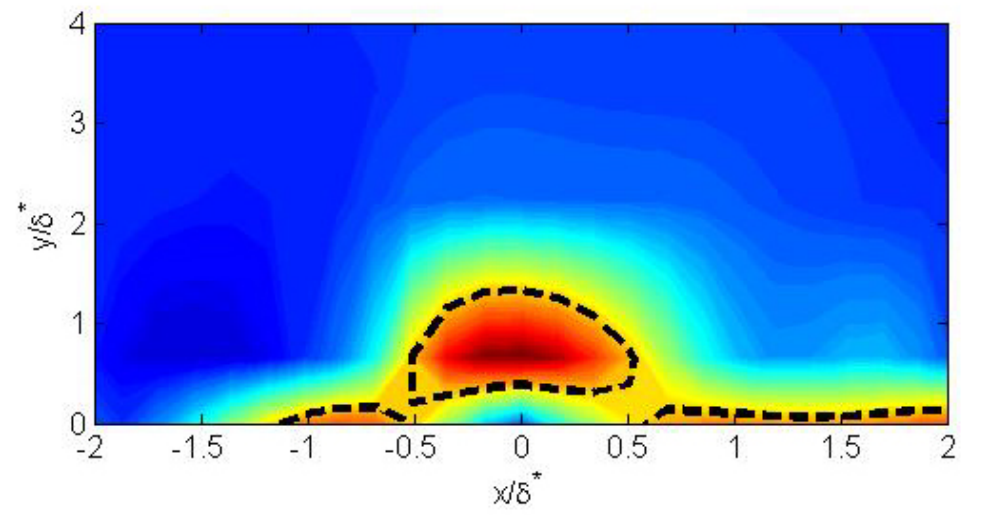

Figure 10. Contour plot of $-\lambda_{2}$, used to identify coherent vortical structures

The $-\lambda_{2}$ criteria was applied to the conditionally sampled velocity field, presented in Figure 9. The velocity gradient field was calculated using a first order difference scheme, with 201 points in the streamwise direction and 5 points in the wall normal direction (four x-wire locations plus imposing the noslip condition at the wall). Due to the small physical size of the boundary layer under investigation $(\delta=1.9 \mathrm{~cm})$, only four $\mathrm{x}$-wires were able to fit into the boundary layer.

The result from applying the $-\lambda_{2}$ criteria is shown in Figure 10. Regions of red indicate an area of $-\lambda_{2}>0$ denoting a coherent vortical structure. Regions of blue indicate areas of stress-dominated flow, and have no significance. The dashed black line was added to highlight the presence of a coherent vortical structure above the optical measurement location.

The coherent vortical structure extended $\sim \delta^{*}$ in the streamwise direction and $\sim 1.2 \delta^{*}$ in the wall normal direction, and is centered at a wall normal distance of $\sim \delta^{*}$. These length scales are in agreement 
with the length scales reported in Reference 10, confirming, with direct evidence, that large scale, coherent vortical structures are responsible for the optical aberrations in turbulent boundary layers.

Length scales measured in the wall normal direction from the $\lambda_{2}$-technique should be lightly regarded due to the fact that there are only 5 measurement points (including an imposition of the no slip condition at the wall) in the wall normal direction, and the $\lambda_{2}$-technique relies on differentiation in both the wall normal and streamwise directions. This accuracy of the wall normal differentiation, and thus the length scale, could be improved, of course, by a better spatial resolution (more $\mathrm{x}$-wire probes). For the current case, it was not possible to achieve a better spatial resolution because the portion of the boundary layer under investigation was $2 \mathrm{~cm}$ thick, and the probe support would not allow for more probes in such a small space.

Recalling the length scales measured in the correlation length experiment ${ }^{8}$, the streamwise optical length scale was measured to be $\sim 2 \delta^{*}$, and from surface pressure measurements to be $\sim 1.5 \delta^{*}$. Comparing these to the length scale of the coherent structure identified by the $\lambda_{2}$ criteria, these measured length scales are in agreement. The length scale in the wall normal direction ${ }^{8}$, obtained from streamwise velocity correlations, was $\sim 1.3 \delta^{*}$. This length scale agrees with the length scale of the identified coherent structure from the conditional average.

\section{Conclusions and Discussions}

The indirect evidence presented in Refs. 7 and 8 pointed to the pressure and density wells associated with large scale, coherent, vortical structures as the physical mechanism for the optical aberrations in turbulent boundary layers. This indirect evidence was based on a high convective speed of optically aberrating flow structures ( $80 \%$ of the freestream), a simple vortical structure model that correctly predicted level of optical aberrations in a turbulent boundary layer scale with density, the boundary layer thickness and the Mach number squared, as well as large, on the order of the boundary layer thickness, correlation lengths of optical, surface pressure, and velocity measurements. All of these are strong indicators that coherent vortical structures in the outer portion of the boundary layer are responsible for optical aberrations.

Cross correlations of simultaneously measured surface pressure, velocity, and optical aberrations showed that the maximum correlations occurred at wall normal distances of less than $2 \delta^{*}$. The characteristic streamwise separation between optically active flow structures was found to be $\sim 7 \delta^{*}$. The spectral correlation between surface pressure and optical aberrations, which shows a relatively high degree of correlation at low frequencies, excludes the possibility that it is small scale turbulence near the wall that is responsible for optical aberrations, as was thought earlier based on the analysis of the aero-optic linking equation ${ }^{1}$. These cross-correlation measurements further suggest that it is coherent structures in the outer portion of the boundary layer that are responsible for optical aberrations.

The conditional sampling algorithm and conditional averaging presented in the paper has confirmed, with direct evidence, that the pressure and density wells associated with coherent, vortical structures are responsible for optical aberrations in turbulent boundary layers. The conditionally averaged surface pressure showed a well defined pressure drop on the surface over the optical measurement location, which is an indicator of the presence of a vortical structure. The conditionally averaged velocity field showed a swirling motion consistent with that of a vortical structure in the outer portion of the turbulent boundary layer, although more resolved in the wall-normal direction measurements are needed.

The $-\lambda_{2}$ criteria, applied to the conditionally sampled velocity data showed a well defined, coherent, vortical structure was present above the optical measurement location. This structure extended $\sim \delta^{*}$ in the streamwise direction and $\sim 1.2 \delta^{*}$ in the wall normal direction, and was centered at a wall normal distance of $\sim 1 \delta^{*}$. These length scales are consistent with the length scales reported in Ref. 8 , lending additional credibility to the length scales reported in that work.

The surface pressure signal showed a well defined pressure well, and the correlation between surface pressure and optical aberrations occurred at relatively low frequencies $f<5 \mathrm{kHz}$, suggesting that it may be possible to predict optical aberrations from surface pressure measurements, and use a currently available adaptive optics system to correct the optical aberrations imparted by a turbulent boundary layer.

Page 9 of 10

American Institute of Aeronautics and Astronautics 


\section{Acknowledgments}

These efforts were sponsored by the Air Force Office of Scientific Research, Air Force Material Command, USAF, under Grant Number F49620-03-1-0019. The U.S. Government is authorized to reproduce and distribute reprints for governmental purposes notwithstanding any copyright notation thereon. This work was completed prior to April, 1, 2005.

\section{References}

${ }^{1}$ Jumper, E. J. and Fitzgerald, E. J., "Recent Advances in Aero-Optics," Progress in Aerospace Sciences, Vol. 37, 2001, pp. 299-339.

${ }^{2}$ Fitzgerald, E. J. and Jumper, E .J., "The Optical Distortion Mechanism in a Nearly Incompressible Free Shear Layer," Journal of Fluid Mechanics, Vol. 512, 2004, pp. 153-189.

${ }^{3}$ Robinson, S., "Coherent motions in the turbulent boundary layer," Annual Review of Fluid Mechanics, Vol. 23, 1991, pp. 601-639.

${ }^{4}$ Christensen, K., and Adrian, R., "The Velocity and Acceleration Signatures af Small-Scale Vortices in Turbulent Channel Flow," In Proceedings of the 2nd International Symposium on Turbulence and Shear Flow Phenomenon, June 2001.

${ }^{5}$ Dargahi, B., "Generation of Coherent Structures in Turbulent Boundary Layers," Journal of Engineering Mechanics, Vol. 123, 1997, p. 7.

${ }^{6}$ Tromeur, E., Garnier, E., Sagaut, P., and Basdevant, C., "Large Eddy Simulations of Aero-Optical Effects in a Turbulent Boundary Layer," Journal of Turbulence, Vol. 4, 2003, pp. 1-22.

${ }^{7}$ Gordeyev, S., Jumper, E. J., Cain, A. B., and Ng, T. T., "Aero-Optical Characteristics of Compressible, Subsonic, Turbulent Boundary Layers," AIAA Paper 2003-3606, Jan. 2003.

${ }^{8}$ Buckner, A., Gordeyev, S., and Jumper, E.J., "Optical Aberrations Caused by Transonic Attached Boundary Layers: Underlying Flow Structure,” AIAA Paper 2005-0752, Jan. 2005.

${ }^{9}$ Buckner, A., "Optical Aberrations in a Subsonic, Compressible, Turbulent Boundary Layer,", M.S. Thesis, University of Notre Dame, September, 2005.

${ }^{10}$ Malley, M., Sutton, G.W., and Kincheloe, N., "Beam-Jitter Measurements for Turbulent Aero-Optical Path Differences, Applied Optics, Vol 31, 1992, pp. 4440-4443.

${ }^{11}$ Ganapathisubramani, B., Longmire, E., and Marusic, I., "Characteristics of Vortex Packets in Turbulent Boundary Layers," Journal of Fluid Mechanics, Vol. 478, 2003, pp. 35-46.

${ }^{12}$ Jeong, J. and Hussain, F., "On the Identification of a Vortex," Journal of Fluid Mechanics, Vol. 285, 1995, pp. 69-94.

Page 10 of 10

American Institute of Aeronautics and Astronautics 